\title{
Protein Cycling Pathway
}

National Cancer Institute

\section{Source}

National Cancer Institute. Protein Cycling Pathway. NCI Thesaurus. Code C40421.

A representation of the sequence of biological or biochemical events that occur as a protein is cycled between the plasma membrane and endosomal vesicles. 